\title{
Yield stress discontinuity in a simple glass
}

\author{
F. Varnik ${ }^{1}$ and O. Henrich ${ }^{2}$ \\ ${ }^{1}$ Max-Planck Institut für Eisenforschung, Max-Planck Straße 1, 40237 Düsseldorf, Germany \\ ${ }^{2}$ Fachbereich für Physik, Universität Konstanz, 78457 Konstanz, Germany
}

(Received 29 November 2005; published 24 May 2006)

\begin{abstract}
Large-scale molecular-dynamics simulations are performed to study the steady-state yielding dynamics of a well-established simple glass. In contrast to the supercooled state, where the shear stress, $\sigma$, tends to zero at vanishing shear rate, $\dot{\gamma}$, a stress plateau forms in the glass which extends over about two decades in shear rate. This strongly suggests the existence of a finite dynamic yield stress in the glass, $\sigma^{+}(T) \equiv \sigma(T ; \dot{\gamma} \rightarrow 0)>0$. Furthermore, the temperature dependence of $\sigma^{+}$suggests a yield stress discontinuity at a critical temperature of $T_{\mathrm{c}}=0.4 \mathrm{in}$ agreement with recent mode coupling theory predictions. The corresponding qualitative change of the flow curves enables us to bracket the critical temperature $T_{\mathrm{c}}$ of the theory from above and from below. We scrutinize and support this observation by testing explicitly for the assumptions (affine flow, absence of flow-induced ordering) inherent in the theory. Furthermore, while qualitative similarity is found between the viscosity and the final relaxation time of stress fluctuations, significant quantitative differences are observed in the nonlinear regime.
\end{abstract}

PACS number(s): 64.70.Pf, 05.70.Ln, 83.60.Df, 83.60.Fg

Soft glassy materials under shear exhibit a rich phenomenology. In the dilute regime, at temperatures corresponding to the liquid state, forced Rayleigh scattering experiments ${ }^{1}$ show an increase of the diffusion constant upon shearing (shear thinning). At higher densities, experiments show evidence for shear thinning due to the presence of freely slipping two-dimensional crystalline layers. ${ }^{2}$

On the other hand, studies of disordered suspensions of hard spheres show that shear thinning and shear melting phenomena may also occur in the absence of an ordered structure. ${ }^{3}$ Similar observations have also been made in lightscattering echo studies of (disordered) dense emulsions. ${ }^{4}$ Brownian dynamics simulations show that shear thinning in concentrated colloidal suspensions is related to the fact that, in the limit of low shear rates, the main contribution to the shear stress originates from the Brownian motion of colloidal particles and that this contribution decreases with shear rate $\dot{\gamma}$ (Ref. 5).

Recently, Berthier et al. studied numerically a driven spin glass and showed that shear thinning can be understood in terms of an acceleration of inherently slow system dynamics by the external drive. ${ }^{6}$ Within this approach, the glassy stress depends on the shear rate via a power law (no dynamical yield stress). The "soft glassy rheology model" (SGR) of Sollich et $a .^{7}$ extends the minimal "trap model" originally introduced by Bouchaud ${ }^{8}$ in order to take into account the effect of an external drive. The theory contains a noise temperature, $x$, which controls the distance from the glass transition at $x=1$. For $1<x<2$, a power-law decrease of the stress with applied shear rate is found, whereas in the jammed state $(x<1)$, a continuous onset of a dynamic yield stress is predicted, $\sigma^{+} \equiv \sigma(\dot{\gamma} \rightarrow 0)=1-x$ (within the SGR, both the dynamic yield stress and the effective noise temperature are expressed in dimensionless units). Starting from the well-studied mode coupling theory (MCT) of the glass transition, ${ }^{9}$ Fuchs and Cates ${ }^{10}$ build upon the idealized picture that, in a supercooled liquid, nearest neighbors of a particle form a cage which progressively solidifies, eventually leading to a complete arrest of all particles as the glass transition is reached. This defines the (ideal) glass transition of MCT, and the temperature at which this transition is predicted is called the mode coupling critical temperature, $T_{\mathrm{c}}$. The effect of shear then enters by the advection of density fluctuations. Fluctuations of a given length scale are advected towards progressively shorter length scales so that particles need explore smaller regions in order for density correlations to decay. The interesting prediction of a yield stress discontinuity at the (ideal) glass transition was made. A related MCT approach to the fluctuations around the steady state has recently been proposed by Miyazaki and Reichman. ${ }^{11}$ The issue of yield stress discontinuity, however, could not be addressed in that approach.

The present work is largely motivated by these qualitatively differing predictions about steady-state flow curves of sheared glasses. Large-scale molecular-dynamics simulations are performed to study the steady-state yielding dynamics of a well-established simple glass.

Berthier and Barrat $^{6}$ performed molecular-dynamics simulation studies of the present model (see below) under a homogeneous shear showing, e.g., that, in a range of low shear rates, time-shear superposition and space-time factorization theorems hold, thus suggesting that generic properties related to the glass transition "generalize" to the nonequilibrium situation of a homogeneous shear. However, due to a rather limited range of shear rates, results presented in Ref. 6 did not allow a clear answer as to whether the present model exhibits a yield stress or not.

In this paper, we focus exactly on this aspect, namely an analysis of the dynamic yield stress and its behavior at the (ideal) glass transition. For this purpose, we performed molecular-dynamics simulations of a generic glass former, a 80:20 binary mixture of Lennard-Jones (LJ) particles (whose types we call A and B) at a constant total density of $\rho=1.2$ in a cubic box of length $L=10(N=1200$ particles $)$. A and B particles interact via $U_{\mathrm{LJ}}(r)=4 \epsilon_{\alpha \beta}\left[\left(d_{\alpha \beta} / r\right)^{12}-\left(d_{\alpha \beta} / r\right)^{6}\right]$, with $\alpha, \beta=\mathrm{A}, \mathrm{B}, \epsilon_{\mathrm{AB}}=1.5 \epsilon_{\mathrm{AA}}, \epsilon_{\mathrm{BB}}=0.5 \epsilon_{\mathrm{AA}}, d_{\mathrm{AB}}=0.8 d_{\mathrm{AA}}$, 
$d_{\mathrm{BB}}=0.88 d_{\mathrm{AA}}$, and $m_{\mathrm{B}}=m_{\mathrm{A}}$. The potential was truncated at twice the minimum position of the LJ potential, $r_{\mathrm{C}}=2.245$. The parameters $\epsilon_{\mathrm{AA}}, d_{\mathrm{AA}}$, and $m_{\mathrm{A}}$ define the units of energy, length, and mass. All other quantities reported in this paper are expressed as a combination of these units. Equations of motion are integrated using a discrete time step of $d t$ $=0.005$.

Equilibrium properties of this model system have been studied extensively in Ref. 12. In particular, it is shown that the equilibrium dynamics of the model can be well described by the "ideal" MCT (Ref. 9) with a critical temperature of $T_{\mathrm{c}}=0.435$.

Results are averaged over ten independent runs. For this purpose, ten independent samples are equilibrated at a temperature of $T=0.45$ (above $T_{\mathrm{c}}$ ) and serve as starting configurations for all simulated temperatures and shear rates. The temperature is controlled via Nosé-Hoover thermostat. ${ }^{13}$ It is set from $T=0.45$ to the desired value at the beginning of shear, whereby only the $y$ component of particle velocities is coupled to the heat bath ( $x$ being the streaming and $z$ the shear gradient directions; see also below).

The temperature quench is done only in one step, i.e., without a continuous variation from $T_{\text {start }}$ to $T_{\text {end }}$. However, as the numerical value of $T$ is changed, it takes a time of the order of the velocity autocorrelation time for the new temperature to be established. During this period of time the Maxwell distribution of velocities undergoes changes in order to adapt itself to a distribution determined by the new temperature. This time is of order unity (in reduced units) and quite short compared to all other relevant time scales in the problem.

Previous studies of the stress-strain relation of the same model showed that the initial transient behavior is limited to strains below $50 \%$ (Ref. 14). Indeed, by shifting the time origin in measurements of various correlation functions, we verified that the time translation invariance was well satisfied in sheared systems for strains larger than $50 \%$. We neglected strains $\gamma<100 \%$ before starting the measurements. Unless otherwise stated, all simulations reported below had a length corresponding to a strain of $7.8(780 \%)$. In the steady state, correlation functions were averaged both over independent runs and over time origins distributed equidistantly along each simulation run. The shear stress is calculated using the virial expression ${ }^{15} \sigma=1 / L^{3}\left\langle\sum_{i}^{N} v_{i x} v_{i z}+\frac{1}{2} \sum_{i \neq j}^{N} x_{i j} F_{z i j}\right\rangle$, where $\langle\cdots\rangle$ stands for statistical averaging, $v_{i}$ is the velocity of $i$ th particle, $x_{i j}=x_{j}-x_{i}$, and $F_{z i j}$ the $z$ component of the force of particle $j$ on $i$.

Recently, it was found that the present model may exhibit shear localization in the glassy state if the shear rate is imposed by using a conventional Couette cell with moving atomistic walls. ${ }^{14}$ However, in the present analysis we are interested in effects of a spatially constant shear rate, a basic ingredient of all theories briefly addressed above. Therefore, we do not use atomistic walls but apply the so-called SLLOD algorithm combined with the Lees-Edwards boundary condition. ${ }^{15}$ With this simulation method, we do indeed observe a linear velocity profile in all studied cases.

Within the SLLOD algorithm, the equations of motion along the streaming direction are modified in order to take the effect of a homogeneous shear into account. In the present case, the streaming velocity is in the $x$ direction while its (linear) variation is along the $z$ direction. As already mentioned, the SLLOD algorithm is combined with the Lees-Edwards boundary condition. The latter consists of moving the periodic image of the simulation cell on the top (bottom) with a constant velocity of $V(-V)$. This naturally defines the shear rate $\dot{\gamma}=V / L$.

In addition to the use of the SLLOD algorithm, the $y$ component of the particles' momenta is coupled to the heat bath in order to impose a constant temperature. This results in the following set of equations for the particle coordinates $(x, y, z)$ and momenta $\left(p_{x}, p_{y}, p_{z}\right)$, solved via discrete integration ( $i$ denotes the particle index, $F$ is the total force on a particle, and $m_{i}$ the particle's mass):

$$
\begin{gathered}
\dot{x}_{i}=p_{x i} / m_{i}+z_{i} \dot{\gamma}, \quad \dot{y}_{i}=p_{y i} / m_{i}, \quad \dot{z}_{i}=p_{z i} / m_{i} \\
\dot{p}_{x i}=F_{x i}-p_{z i} \dot{\gamma}, \quad \dot{p}_{y i}=F_{x i}-\xi p_{y i}, \quad \dot{p}_{z i}=F_{z i} \\
\dot{\xi}=\left(\sum_{i} p_{y i}^{2} / m_{i}-N k_{\mathrm{B}} T\right) / Q .
\end{gathered}
$$

It is noteworthy that the momenta occurring in the above equations are the peculiar ones, i.e., they correspond to the particles' momenta in a (local) frame of reference moving with the flow $(\langle\boldsymbol{p}\rangle=\mathbf{0})$ (Ref. 15).

As also seen from the above equations, the variable $\xi$ plays the role of a friction (acceleration) coefficient, since a positive (negative) $\xi$ tends to decrease (increase) $p_{y}$. The variation of $\xi$, on the other hand, is controlled by the deviation of the actual kinetic energy of the system from that prescribed by the temperature, $T$, of the heat bath. The parameter $Q$ controls the strength of the coupling of the particles' momenta to the heat bath (the smaller $Q$ the stronger the coupling). In previous studies, it is found that an optimum choice for $Q$ exists: ${ }^{16} Q=6 N k_{\mathrm{B}} T / \omega^{2}$, where $\omega$ is a characteristic vibrational frequency and $k_{\mathrm{B}}(\equiv 1)$ is the Boltzmann constant (see also Ref. 17, and the references therein).

The "optimum choice" of $Q$ is defined via the requirement that the sampling of the system energy is unchanged and corresponds to a unique (canonical ensemble) distribution if $Q$ is varied around this specific value. For the present LJ model, we set $\omega=10.7$, which roughly corresponds to the oscillation frequency at the minimum of the Lennard-Jones potential for the interaction between particles of type A.

Since shear may, at least in principle, change the static structure of the system, we examine this by computing pair distribution functions along $x$ (flow), and $y$ and $z$ (shear gradient) directions separately. For the $x$ direction, for example, we compute the (majority) A-A pair correlation via $g(x)$ $=\left\langle\sum_{i=1}^{N_{\mathrm{A}} \sum_{j>i}} \delta\left(\left|x_{i}-x_{j}\right|-x\right) \delta\left(y_{i}-y_{j}\right) \delta\left(z_{i}-z_{j}\right)\right\rangle$, and finally normalize the result by the ideal gas value so that, in the absence of long-range order, $g(x \rightarrow \infty)=1$ is expected $\left(N_{\mathrm{A}}\right.$ is the number of A particles). Figure 1 illustrates results on $g(x), g(y)$ and $g(z)$ (A-A correlations) for three characteristic temperatures $T=0.2$ (below $T_{\mathrm{c}}$ ), $T=0.42$ (close to $T_{\mathrm{c}}$ ), and $T=0.6$, indicating the absence of long-range order in all studied cases. Surprisingly, even though the stress changes by more than a decade at this $\dot{\gamma}$, the local structure varies little with 


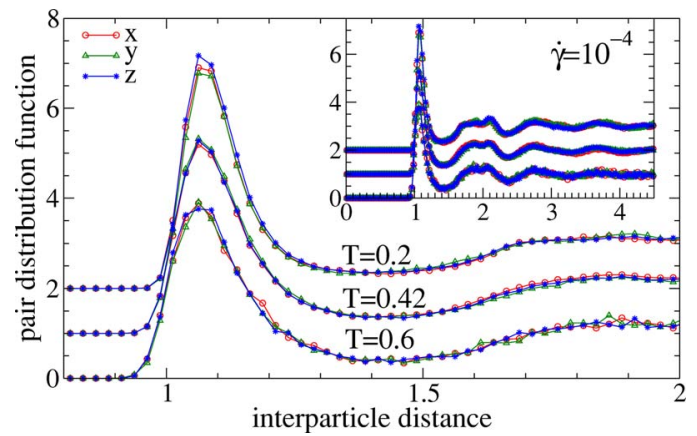

FIG. 1. (Color online) A-A pair correlation functions along $x$ (flow), and $y$ and $z$ (shear gradient) directions, for three characteristic temperatures: $T=0.2$ (glass), $T=0.42$ (close to $T_{\mathrm{c}}$ ), and $T$ $=0.6$ (supercooled state). The shear rate is $\dot{\gamma}=10^{-4}$. For clarity, data at $T=0.42(T=0.2)$ are shifted upwards by $1(2)$.

shear, remains amorphous, and (almost) isotropic. Similar observations are also made using the B-B and A-B pair correlations.

Figure 2 shows simulated steady-state shear stresses as functions of shear rate (viz. "flow curves") for temperatures ranging from far above to far below the critical temperature of the model. As apparent from the change of the curvature of the flow curves (S-shaped without extended horizontal piece at high, horizontal piece merging into upward curvature at low temperatures) the system response changes qualitatively around a critical temperature $T_{\mathrm{c}}$, which MCT identifies as (ideal) glass transition temperature. For $T>T_{\mathrm{c}}$ the stress becomes proportional to the shear rate as $\dot{\gamma}$ approaches zero (linear response). At temperatures below $T_{\mathrm{c}}$, however, a stress plateau forms in the low $\dot{\gamma}$ regime. Importantly, the qualitative change manifest in the $\sigma(\dot{\gamma})$ curves enables us to give upper and lower limits for $T_{\mathrm{c}}$ without any theoretical analysis: we conclude $0.34<T_{\mathrm{c}}<0.45$. A more quantitative test is done by solving mode coupling equations for the schematic $F_{12}^{\dot{\gamma}}$ model. ${ }^{10}$ It consists of an extension of the wellknown $F_{12}$ model. ${ }^{9}$ The normalized correlation function obeys

$$
\dot{\Phi}(t)+\Gamma\left\{\Phi(t)+\int_{0}^{t} d t^{\prime} m\left(t-t^{\prime}\right) \dot{\Phi}\left(t^{\prime}\right)\right\}=0,
$$

where the memory kernel $m$ contains a $\dot{\gamma}$-dependent prefactor to account for the loss of memory during shearing,

$$
m(t)=\frac{1}{1+(\dot{\gamma} t)^{2}}\left[v_{1} \Phi(t)+v_{2} \Phi^{2}(t)\right] .
$$

The vertices $v_{i}$ mimic the cage effect and set the bifurcation points, separating glassy from fluid solutions, $v_{1}^{c}=\sqrt{4 v_{2}^{c}}-v_{2}^{c}$, with a typical choice $v_{2}^{c}=2$.

The choice of $v_{2}^{c}=2$ in the schematic model leads to the same discontinuous type-B transition as in the microscopic description of structural glass formers, and therefore provides a model with all important features. Last, but not least, this specific choice is motivated by the rich variety of previous theoretical studies of the schematic $F_{12}$ model using this

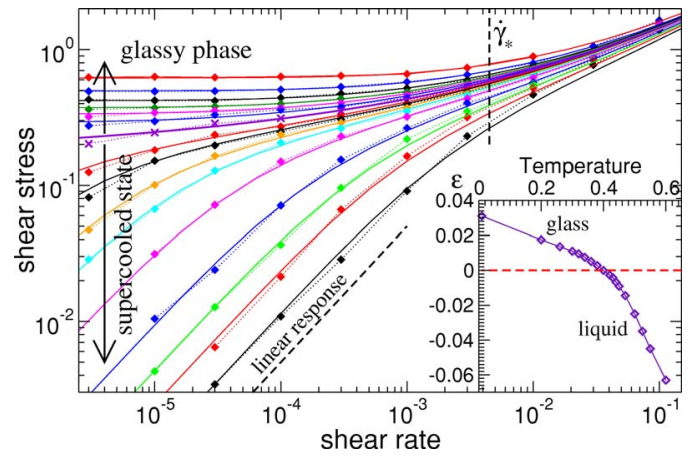

FIG. 2. (Color online) Simulated shear stress (symbols) compared to theoretical model calculations (solid lines) for various temperatures (from top to bottom: $T=0.01,0.2,0.3,0.34,0.36,0.38$, $0.4,0.42,0.43,0.44,0.45,0.47,0.5,0.525,0.55,0.6)$. The inset shows the fitted separation parameter $\epsilon$ vs $T$.

value (see, e.g., Ref. 9, and references therein) which allow for tests and interpretations of the present results in light of the previous works.

To pass the transition line following studies of Ref. 10, $v_{1}$ is increased according to $v_{1}=v_{1}^{c}+\varepsilon /\left(\sqrt{v_{2}^{c}}-1\right)$, while $v_{2}$ is kept fixed. The separation parameter $\varepsilon$ gives the distance from the (ideal) glass transition and distinguishes fluid $(\varepsilon$ $<0)$ from glassy $(\varepsilon \geq 0)$ solutions. Finally, following Maxwell, the viscosity is modeled by the average relaxation time $\eta=\sigma / \dot{\gamma}=\int_{0}^{\infty} d t \Phi(t)$.

It is important to realize that, here, the integration starts at the onset of the shear and thus is stretched over the transient correlations. This is a fundamental difference to a definition, where steady-state correlations are used. While the former yields the physical viscosity, the latter presents a rather $a d$ hoc definition of the viscosity which converges towards its physical counterpart as the linear response regime is approached. As will be shown below, the difference between these two quantities may be quite significant in the glassy state (see the discussion of Fig. 4).

As seen from Fig. 2, fits with the schematic $F_{12}^{\dot{\gamma}}$ model achieve to describe the flow curves for all studied temperatures by adjusting two global parameters (a scale for $\dot{\gamma}$ and one for $\sigma$ ), and the parameter $\varepsilon$ at each temperature measuring the relative distance to the (ideal) glass transition. ${ }^{18}$ The fit gives $T_{\mathrm{c}}=0.4$ and $\sigma_{\mathrm{c}, \mathrm{th}}^{+}=0.19$. We thus conclude that the basic rheological features of our model are well described within simple schematic models in the framework of the idealized MCT. Relaxation channels not contained in the idealized MCT (so-called "hopping effects," 12 which may be the origin for the deviations from theory close to $T_{\mathrm{c}}$ and at very low $\dot{\gamma}$ in Fig. 2) cannot falsify our bound for $T_{\mathrm{c}}$, because the qualitatively different shapes of the flow curves are the characteristics of the fluid or glassy states within MCT.

The stress plateau is best developed for temperatures deep in the glassy phase extending over about two decades in shear rate. Its onset is shifted toward progressively lower $\dot{\gamma}$ as the temperature is increased toward $T_{\mathrm{c}}$. This makes an estimate of the dynamic yield stress, $\sigma^{+}(T) \equiv \sigma(T ; \dot{\gamma} \rightarrow 0)$, a difficult task for temperatures below but close to $T_{\mathrm{c}}$. Nevertheless, an estimate of $\sigma^{+}(T)$ is interesting because it high- 


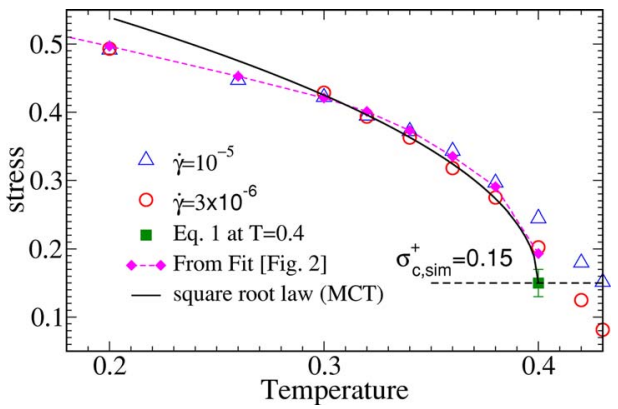

FIG. 3. (Color online) Determination of dynamic yield stress and its temperature dependence (see text).

lights the anomalous weakening of the glass when heating to $T_{\mathrm{c}}$. Testing the MCT predictions below $T_{\mathrm{c}}$ has previously not been possible in simulations because of problems to reach the equilibrated or steady state at sufficiently low shear rates. Our estimate is obtained by comparing the steady-state shear stress for the two lowest simulated shear rates, namely $\dot{\gamma}$ $=10^{-5}$ and $\dot{\gamma}=3 \times 10^{-6}$. As shown in Fig. 3, at temperatures below $T=0.38$, practically the same shear stress is obtained for both choices of $\dot{\gamma}$ indicating the presence of a yield stress plateau.

For $T=T_{\mathrm{c}}$, we make use of theoretical predictions based on the $F_{12}^{\dot{\gamma}}$ model. ${ }^{18}$ For not too low shear rates, the flow curve takes the form of a generalized Hershel-Bulkeley constitutive equation,

$$
\sigma=\sigma_{\mathrm{c}}^{+}\left(1+\left|\dot{\gamma} / \dot{\gamma}_{*}\right|^{m}+c_{2}\left|\dot{\gamma} / \dot{\gamma}_{*}\right|^{2 m}+c_{3}\left|\dot{\gamma} / \dot{\gamma}_{*}\right|^{3 m}\right) .
$$

Here, $\dot{\gamma}_{*}$ is an upper limit where this expansion holds, and the exponent $m$ is connected to the familiar exponent parameter $\lambda$ of MCT (Refs. 9 and 10). As the simple $F_{12}^{\dot{\gamma}}$ model is found to describe the rheological properties of our system rather well (see the discussion of Fig. 2), we set the parameters $c_{2}=0.896, c_{3}=0.95$, and $m=a(2 \lambda-1) /(1+a) \lambda=0.143$ (with $a=0.324$ ) as obtained in the model. ${ }^{18} \mathrm{We}$ apply a fit to Eq. (1) with $\sigma_{\mathrm{c}}^{+}$and $\dot{\gamma}_{*}$ being the only fit parameters. This gives $\sigma_{\text {c,sim }}^{+}=0.15 \pm 0.01$ and $\dot{\gamma}_{*}=0.0045 \pm 0.0008$, the latter being close to our estimate of the window, where MCT can describe the flow curves. At higher shear rates, $\dot{\gamma}>\dot{\gamma}_{*}$, we expect microscopic effects to dominate the stress. As shown in Fig. 3, while $\sigma^{+}(T)$ weakly varies with $T$ at low temperatures, it steeply drops as $T$ approaches $T_{\mathrm{c}}$, signaling the (ideal) glass transition. The yield stress follows well the MCT square-root law, ${ }^{10} \sigma^{+}(T)-\sigma_{\mathrm{c}}^{+} \propto\left|1-T / T_{\mathrm{c}}\right|^{0.5}$.

Bear in mind that the critical temperature of $T_{\mathrm{c}}=0.4$, used in order to obtain best agreement between the theory and simulations, is slightly lower than the estimate $T_{\mathrm{c}}=0.435 \mathrm{ob}-$ tained from the analysis of the equilibrium dynamics of the system. ${ }^{12}$ This discrepancy is possibly related to the hopping effects observed in the density correlation functions closely above $T_{\mathrm{c}}$ (Refs. 12 and 19). A closer analysis of this aspect requires understanding of hopping effects under shear and is beyond the scope of the present report.

Next we define a stress via an ad hoc nonequilibrium Green-Kubo relation, $\hat{\sigma}=\dot{\gamma} V /\left(k_{\mathrm{B}} T\right) \int_{0}^{\infty}\left\langle\sigma_{x y}(t) \sigma_{x y}(0)\right\rangle d t(V$ denotes the system volume) employing the stress fluctuations

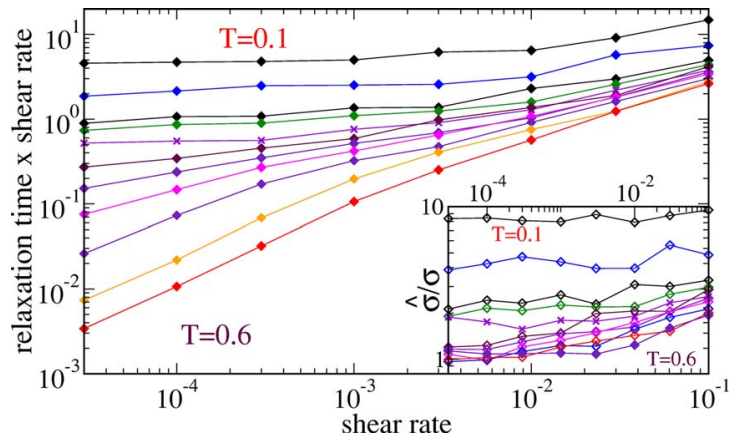

FIG. 4. (Color online) $\hat{\sigma} \equiv \dot{\gamma} V /\left(k_{\mathrm{B}} T\right) \int_{0}^{\infty} d t\left\langle\sigma_{x y}(t) \sigma_{x y}(0)\right\rangle$ vs shear rate for various temperatures ranging from the glassy phase to the supercooled state (from top to bottom: $T=0.1,0.2,0.3,0.34,0.4$, $0.42,0.45,0.47,0.5,0.55,0.6)$. The inset shows the ratio of this stress to the real shear stress.

around the steady state. Note that, in our simulations, the nonzero component of the stress tensor is $\left\langle\sigma_{x z}\right\rangle$, whereas $\left\langle\sigma_{x y}\right\rangle=0$ so that the integrand decays to zero at large times. In order to determine stress autocorrelation (and thus $\hat{\sigma}$ ) accurately, we performed very long simulations up to a strain of 100 (while averaging over time origin). For this reason, $\hat{\sigma}$ could be determined with a reasonable accuracy only for shear rates $\dot{\gamma} \geq 3 \times 10^{-5}$ (see Fig. 4).

As a comparison of Figs. 2 and 4 reveals, there is a strong similarity between the $\dot{\gamma}$ dependence of $\hat{\sigma}$ and that of the shear stress. As seen from the inset of Fig. 4, the quotient $\hat{\sigma} / \sigma$ tends to unity for temperatures above $T_{\mathrm{c}}$ in the limit of low shear rates. This behavior indicates the linear response regime. In the glassy state, however, a large gap opens between $\hat{\sigma}$ and $\sigma$ at all shear rates. At a temperature of $T$ $=0.1$, for example, $\hat{\sigma}$ is by approximately a factor of 8 larger than $\sigma$, even in the limit of $\dot{\gamma} \rightarrow 0$.

In summary, large-scale molecular-dynamics simulations have been performed in order to investigate the existence and temperature dependence of the dynamic yield stress, $\sigma^{+}$, for a 80:20 binary Lennard-Jones model first proposed by Kob and Andersen. ${ }^{12}$ Our data do indeed support the existence of a dynamic yield stress in the glassy phase as underlined by stress plateaus extending over about two decades in shear rate. Let us mention recent experiments on the rheology of dense colloidal dispersions ${ }^{20}$ which also find finite $\sigma^{+}$. Furthermore, the temperature dependence of $\sigma^{+}$follows the predicted anomalous weakening close to the critical temperature of MCT. We estimate a finite critical yield stress of $\sigma_{\mathrm{c}}^{+}$ $=0.17 \pm 0.02$. The flow curves allow for the first bracketing in simulations of the critical temperature of MCT from below. Irrespective of hopping effects neglected in the employed MCT, we can conclude $T_{\mathrm{c}}>0.34$.

Furthermore, a generalized stress ( $\hat{\sigma}$; Fig. 4$)$ based on the stress fluctuations around the steady state was determined for all temperatures and shear rates. $\hat{\sigma}$ exhibits the same qualitative features as the real shear stress, $\sigma$, thus emphasizing the close connection between the fluctuations and the rheological response. However, beyond the linear response regime, $\hat{\sigma}$ increases much faster than $\sigma$ as temperature is decreased. This observation is quite significant as it concerns 
theoretical approaches where, even beyond the Newtonian regime, the shear viscosity is simply taken as a relaxation time, ${ }^{6,11}$ or where this relation holds as approximation. ${ }^{10}$

We thank J.-L. Barrat, L. Berthier, L. Bocquet, M. E.
Cates, M. Fuchs, and J. Horbach for useful discussions. F.V. was supported by DFG Grant No. VA 205/3-2, and O.H. by the DFG IGC "Soft Matter." Simulation time was granted by the ZDV-Mainz and PSMN-Lyon and IDRIS (Project No. 031668-CP: 9).
${ }^{1}$ X. Qiu, H. D. Ou-Yang, D. J. Pine, and P. M. Chaikin, Phys. Rev. Lett. 61, 2554 (1988).

${ }^{2}$ B. J. Ackerson and N. A. Clark, Phys. Rev. Lett. 46, 123 (1981); B. Ackerson, J. Rheol. 34, 553 (1990).

${ }^{3}$ G. Petekidis et al., Physica A 306, 334 (2002); G. Petekidis, A. Houssaid, and P. N. Pusey, Phys. Rev. E 66, 051402 (2002); G. Petekidis, D. Vlassopoulos, and P. Pusey, Faraday Discuss. 123, 287 (1999); H. M. Laun, R. Bung, S. Hess, W. Loose, O. Hess, K. Hahn, E. Hädicke, R. Hingmann, F. Schmidt, and P. Lindner, J. Rheol. 36, 743 (1992).

${ }^{4}$ P. Hébraud, F. Lequeux, J. P. Munch, and D. J. Pine, Phys. Rev. Lett. 78, 4657 (1997).

${ }^{5}$ T. Phung, J. Brady, and G. Bossis, J. Fluid Mech. 313, 181 (1996); P. Strating, Phys. Rev. E 59, 2175 (1999).

${ }^{6}$ L. Berthier, J.-L. Barrat, and J. Kurchan, Phys. Rev. E 61, 5464 (2000); L. Berthier and J.-L. Barrat, J. Chem. Phys. 116, 6228 (2002); L. Berthier, J. Phys.: Condens. Matter 15, S933 (2003).

${ }^{7}$ P. Sollich, F. Lequeux, P. Hébraud, and M. E. Cates, Phys. Rev. Lett. 78, 2020 (1997); P. Sollich, Phys. Rev. E 58, 738 (1998).

${ }^{8}$ J. Bouchaud, J. Phys. I 2, 1705 (1992).

${ }^{9}$ W. Götze, in Les Houches 1989, Session LI, edited by J. P. Hansen, D. Levesque, and J. Zinn-Justin (North-Holland, Amsterdam, 1989), pp. 287-503.

${ }^{10}$ M. Fuchs and M. E. Cates, Phys. Rev. Lett. 89, 248304 (2002);
Faraday Discuss. 123, 267 (2003); J. Phys.: Condens. Matter 17, S1681 (2005).

${ }^{11}$ K. Miyazaki and D. Reichman, Phys. Rev. E 66, 050501(R) (2002); K. Miyazaki, D. Reichman, and R. Yamamoto, ibid. 70, 011501 (2004).

${ }^{12}$ W. Kob and H. C. Andersen, Phys. Rev. Lett. 73, 1376 (1994); Phys. Rev. E 51, 4626 (1995); 52, 4134 (1995); T. Gleim, W. Kob, and K. Binder, Phys. Rev. Lett. 81, 4404 (1998).

${ }^{13}$ S. Nosé, Mol. Phys. 52, 255 (1984); 57, 187 (1986); W. G. Hoover, Phys. Rev. A 31, 1695 (1985); 34, 2499 (1986).

${ }^{14}$ F. Varnik, L. Bocquet, J.-L. Barrat, and L. Berthier, Phys. Rev. Lett. 90, 095702 (2003); F. Varnik, L. Bocquet, and J.-L. Barrat, J. Chem. Phys. 120, 2788 (2004).

${ }^{15}$ D. J. Evans and G. P. Morriss, Statistical Mechanics of Non Equilibrium Liquids (Academic, London, 1990).

${ }^{16}$ S. Nosé, Prog. Theor. Phys. 103, 1 (1991).

${ }^{17}$ F. Varnik, Ph.D. thesis (2000), available from http:// ArchiMeD.uni-mainz.de/pub/2001/0007/

${ }^{18}$ O. Henrich, F. Varnik, and M. Fuchs, J. Phys.: Condens. Matter 17, 3625 (2005).

${ }^{19}$ E. Flenner and G. Szamel, Phys. Rev. E 72, 011205 (2005).

${ }^{20}$ G. Petekidis, D. Vlassopoulos, and P. N. Pusey, J. Phys.: Condens. Matter 16, S3955 (2004); M. Fuchs and M. Ballauff, J. Chem. Phys. 122, 094707 (2005). 